\title{
Human Umbilical Cord Mesenchymal Stem Cell (HUCMSC)-derived Exosomes as a Cell-Free Therapy for Soluble Fms-like Tyrosine Kinase-1 (Sflt- 1)-induced Endothelial Dysfunction in Preeclampsia
}

XINWEN CHANG ( $\nabla$ cissy917@126.com )

Tongji University School of Medicine

QIZHI HE

Tongji University School of Medicine

MINGMIN WANG

Tongji University School of Medicine

LINYAN JIA

TONGJI UNIVERSITY SCHOOL OF MEDICINE

TAO DUAN

Tongji University School of Medicine

KAI WANG

FSBI Ural Research Institute of Maternity and Infant Protection: FGBU Ural'skij naucno-issledovatel'skij institut ohrany materinstva i mladencestva

\section{Research Article}

Keywords: Preeclampsia, mesenchymal stem cell, exosomes, angiogenesis, soluble fms-like tyrosine kinase-1/sFlt-1.

Posted Date: May 5th, 2021

DOI: https://doi.org/10.21203/rs.3.rs-455719/v1

License: (c) (i) This work is licensed under a Creative Commons Attribution 4.0 International License. Read Full License 


\section{Abstract}

\section{Background}

Preeclampsia is a unique multisystem disorder that affects $5-8 \%$ of pregnancies. A high level of soluble fms-like tyrosine kinase-1 (sFlt-1) is a hallmark of preeclampsia that causes endothelial dysfunction. Exosomes derived from mesenchymal stem cells (MSCs) have been indicated to improve endothelial performances by transporting signals to target cells. We hypothesized that exosomes derived from MSCs have potential effects against preeclampsia.

\section{Methods}

We collected human umbilical cord MSC-derived exosomes (HUCMSC-exos) by ultracentrifugation. The size and morphology of the exosomes were examined using a transmission electron microscope and nanoparticle tracking analysis. Pregnant mice were injected with murine sFlt-1 adenovirus to build the preeclampsia-like mouse model and then treated with HUCMSC-exos. Human umbilical vein endothelial cells (HUVECs) were infected with lentiviruses expressing tet-on-sFlt-1 to obtain cells overexpressing sFlt1. Cell proliferation and migration assays were used to measure the endothelial functions. The exosomes enriched proteins underlying mechanisms were explored by proteomic analysis.

Results

In the current study, we successfully collected the cup-shaped HUCMSC-exos with diameters of 30-150 $\mathrm{nm}$. In the sFIt-1-induced preeclampsia mouse model, HUCMSC-exos exhibited beneficial effects on adverse birth events by decreasing blood pressure and improving fetal birth weight. In addition, preeclamptic dams that were injected with HUCMSC-exos had rebuilt dense placental vascular networks. Furthermore, we observed that HUCMSC-exos partially rescued sFlt-1-induced HUVECs dysfunction in vitro. Proteomics analysis of HUCMSC-exos displayed functional enrichment in biological processes related to vesicle-mediated transport, cell communication, cell migration, and angiogenesis.

Conclusion

We propose that exosomes derived from HUCMSCs play beneficial roles in the birth outcomes of sFlt-1induced preeclamptic mice by promoting angiogenesis.

\section{Introduction}

Preeclampsia (PE) is characterized by new-onset hypertension at $\geq 20$ weeks of gestation with or without proteinuria[1]. This gestation-specific syndrome is a serious pregnancy complication that remains one of the main causes of maternal, fetal, and neonatal mortality globally and is also related to intrauterine growth retardation (IUGR) and preterm birth[2]. PE is primarily a consequence of an imbalance between proangiogenic and antiangiogenic growth factors, such as soluble fms-like tyrosine kinase-1 (sFlt-1), 
which causes multisystem endothelial dysfunction[3]. Women who are diagnosed with PE have decreased quality of life and elevated risk of postpartum depression.

Mesenchymal stem cells (MSCs) are defined as multipotent adult cells with the ability to differentiate into multiple cell types. Human umbilical cord MSCs (HUCMSCs) have become a prominent stem cell type used for allogeneic cell-based therapy because of their advantages in ethical access and rapid renewal properties[4]. In addition, HUCMSCs are considered nonimmunogenic because they express low levels of major histocompatibility complex-II (MHC-II) and have therefore emerged as one of the most promising treatment options for vascular diseases[5]. HUCMSCs have been under investigation in a variety of clinical therapeutic trials including cardiovascular deficits, recovering ovarian function, and immune system diseases. Our previous work indicated that HUCMSCs provided a new avenue for treating placenta-related diseases during pregnancy like PE[6], however, the underlying molecular mechanism remains unknown.

A large body of evidence indicates that HUCMSCs secrete extracellular vesicles containing a number of functional molecules. Exosomes are nanosized extracellular vesicles $(30-150 \mathrm{~nm})$ of endocytic origin that are released by most cells[7]. They are cup-shaped phospholipid nanocarriers functioning as transmitting amounts of bioactive molecules for intercellular communication. Exosomes have been well studied in angiogenesis, and our previous research indicated that exosomes from PE patients induced endothelial dysfunction by transferring high levels of sFIt-1 and soluble Endoglin to human umbilical vein endothelial cells (HUVECs)[8]. Therapies with the potential to prevent PE progression or reverse diseaseinduced organ damage are lacking; thus, the pursuit of a treatment option to replace expectant management and instant delivery needs to be investigated. Recently, HUCMSC-derived exosomes (HUCMSC-exos) showed high translational value in anti-aging intervention by enhancing the regenerative capacities in bone formation, wound healing, and angiogenesis[9]. In addition, HUCMSC-exos have been recognized as new candidates for the treatment of vascular diseases because they are selectively enriched in proangiogenic molecules[10]. However, the roles and underlying mechanisms of HUCMSCexos in the treatment of reproductive complications such as PE are only beginning to be understood and appreciated.

Here, we provide new evidence that HUCMSC-exos have the ability to reverse sFlt-1-induced PE-like adverse birth outcomes in vivo. In addition, exosomes can facilitate sFIt-1-imparied endothelial dysfunction in HUVECs. The proteomic analysis revealed that HUCMSC-exos were highly enriched in the proteins involved in regulation of cell communication, cell migration, and angiogenesis, as well as vascular endothelial growth factor receptor signaling pathway. Based on the improvements brought about by HUCMSC-exos treatment in terms of vascular functions and birth quality in PE-like mice model, we propose that HUCMSC-exos may represent a new approach to treat or prevent PE.

\section{Materials And Methods}

\section{Isolation of HUCMSCs}


Human umbilical cord tissues were collected from full-term, healthy cesarean births and were donated by consenting mothers at the Department of Obstetrics of Shanghai First Maternity and Infant Hospital from 2018 to 2019. The demographic and clinical characteristics of the participants in this study were reported in our previous study[11]. Umbilical arteries and veins were removed, and the remaining Wharton's jelly was transferred to sterile, ice-cold Hank's balanced salt solution (HBSS, Gibco, Thermo Fisher Scientific, Inc., Waltham, MA, USA) containing antibiotics (streptomycin/penicillin, Gibco). The tissues were washed multiple times with HBSS to remove excess blood and were subsequently cut into small pieces. The pieces were transferred to $10 \mathrm{~cm}^{2}$ dishes with $5 \mathrm{~mL}$ of minimum Eagle's medium alpha (Gibco) containing $10 \%$ fetal bovine serum (FBS, Gibco) and 1\% streptomycin/penicillin and were incubated at 37 ${ }^{\circ} \mathrm{C}$ in $5 \% \mathrm{CO}_{2}$. The explants were left undisturbed for 7-10 days to allow cell migration, and the medium was changed every three days. After 2 weeks, the HUCMSCs (passage 0 ) were grown to $60-70 \%$ confluence and passaged by trypsinization. Sample collection was approved by the Research and Ethics Committee of Shanghai First Maternity and Infant Hospital.

\section{Identification of HUCMSCs by flow cytometry and osteogenic differentiation}

MSC surface marker detection by a Fluorescence-activated cell sorting (FACS) Calibur Flow Cytometer (BD Biosciences, San Jose, CA, USA) was previously shown[11]. Briefly, $1 \times 10^{6}$ passage 3 cells were trypsinized and suspended in $100 \mu \mathrm{L}$ of phosphate buffer saline (PBS). Then, the cells were incubated for 30 min with the following monoclonal antibodies against HUCMSC surface markers: CD45-PE, CD73-APC, CD90-FITC and CD105-PE-Cy7 (BD Biosciences, San Jose, CA, USA).

For osteogenic differentiation, passage 3 cells were cultured in differentiation medium (Gibco) for 3 weeks in six-well plates. After the cells were fixed with 4\% paraformaldehyde (PFA) and stained with alizarin red S (Sigma-Aldrich, Merck, Darmstadt, Germany), the cells were observed by microscopy (Nikon Eclipse Ti, Tokyo, Japan).

\section{Isolation and characteristics of HUCMSC-exos}

HUCMSCs at passages 3-6 were cultured in FBS-free medium with $2 \%$ bovine serum albumin (BSA, Sigma-Aldrich) for $48 \mathrm{~h}$, and then $500 \mathrm{~mL}$ of the supernatant was centrifuged at $3,000 \mathrm{~g}$ for $10 \mathrm{~min}$ to remove cell debris and passed through a $0.22-\mu \mathrm{m}$ filter. The cleared supernatant was ultracentrifuged at $110,000 \mathrm{~g}$ for $70 \mathrm{~min}$ and washed in PBS using the same ultracentrifugation conditions to isolate exosomes. Exosome pellets were suspended in $5 \mathrm{~mL}$ of PBS and stored at $-80^{\circ} \mathrm{C}$.

The size and morphology of the exosomes were examined using a transmission electron microscope (TEM) at the Laboratory of Electron Microscopy (Chinese Academy of Sciences $₫$ Shanghai, China). The size distribution of HUCMSC-exos was determined using nanoparticle tracking analysis (NTA, ZetaView, Particle Metrix, Meerbusch, Germany). These procedures were performed as previously described[8].

\section{Mice}


All mouse experiments were approved by the Department of Laboratory Animal Science, Tongji University. Adult male $(n=40)$ and female $(n=80)$ CD-1 (ICR) mice were purchased from Jackson Laboratories. Animals were housed in a temperature- and humidity-regulated environment with a 12-h light cycle. For a consistent and accurate assessment of the gestational age of mouse embryos, male and female (1:2) mice were pair-housed for one night. Embryonic day 0.5 (E0.5) was designed as the first morning at which a vaginal plug was noted.

Pregnant mice $(n=32)$ were randomly divided into four groups ( $n=8$ per group) and injected with either Cytomegalovirus (CMV)-null adenovirus or murine sFlt-1 adenovirus on E8.5 and HUCMSC-exos (EXO, 100 $\mu \mathrm{g} / \mathrm{dam}$ ) or sterile saline (NS, $100 \mu \mathrm{L})$ on E6.5, E9.5, E12.5 and E15.5 via the tail vein. The groups were designated as follows: (i) control (CTL): pregnant mice administered CMV-null adenovirus and NS; (ii) EXO: pregnant mice administered CMV-null adenovirus and HUCMSC-exos; (iii) sFlt-1: pregnant mice administered sFlt-1 adenovirus and NS; and (iv) sFlt-1+EXO: pregnant mice administered sFlt-1 adenovirus and HUCMSC-exos. In total, there were 4 treatment groups with respect to sFlt-1 overexpression and HUCMSC-exos administration. Blood pressure was noninvasively measured on E18.5 by determining the tail blood volume with a volume pressure recording sensor (CODA System, Kent Scientific, Torrington, CT, USA) that was averaged over a 10-min period. Embryos were harvested on E18.5, and serum, tissues, and urine samples were obtained before euthanasia as described elsewhere[12].

\section{Tissue preparation for histological analysis}

Placental and kidney tissues were fixed with $4 \%$ PFA for $48 \mathrm{~h}$ and processed by conventional procedures. Sections 3-5 $\mu \mathrm{m}$ in thickness were cut from the paraffin-embedded tissues, mounted on poly-L-lysinecoated slides, deparaffinized in xylene, dehydrated in alcohol and then stained with hematoxylin and eosin (H\&E) or periodic acid-methenamine silver (PAS) stain.

\section{Immunohistochemical (IHC) analysis of mouse placental tissues}

For IHC analysis, paraffin sections were deparaffinized and incubated with citrate buffer for antigen retrieval. The slides were then incubated with rabbit anti-CD31 polyclonal antibody (1:100, Abcam, Cambridge, MA, USA) overnight at $4{ }^{\circ} \mathrm{C}$ and developed using the ImmPRESS horseradish peroxidase (HRP) anti-rabbit IgG polymer detection kit (Weiao, Shanghai, China).

\section{Morphological analysis}

Using CD31 as an endothelial marker, the densities, diameters, and areas of fetal blood vessels were analyzed as previously described[13]. Four images per tissue section were taken using a Nikon inverted microscope with a $40 \times$ objective (vascular density) or 100x objective (vascular diameter). For each image, the number of capillaries was counted, and the lumen area was measured using ImageJ imaging analysis software (NIH, Bethesda, MD). Five capillaries per image were randomly selected for diameter measurements by CaseViewer software (3DHISTECH, Ltd., Hungary). 


\section{ELISAs}

ELISAs for mouse sFlt-1 and sEndoglin were performed according to the manufacturer's instructions (R\&D Systems). Urine albumin/creatinine ratio was measured using Urinary Albumin and Creatinine Assay kits (Abnova, Taipei City, Taiwan, China). Briefly, the various samples were diluted 1:2 in dilutions and were incubated in a 96-well plate pre-coated with capture antibodies. The wells were washed and incubated with a secondary antibody conjugated to horseradish peroxidase. Then substrate solution was added, and optical density was determined at $450 \mathrm{~nm}$. The concentrations were calculated using a standard curve of the respective recombinant proteins.

\section{Western blot}

Total protein concentration was measured using the bicinchoninic acid assay (Pierce ${ }^{\circledR}$, Thermo Fisher Scientific, Bonn, Germany). Western blot for expressions in exosomes were performed using CD63 (1:1000, SBI), CD81 (1:1000, SBI), CD9 (1:1000, SBI), sFlt-1 (1:1000, Abcam), Flag (1:2000, Sigma), eNOS (1:1000, CST), Versican (1:1000, Abcam), a-Tublin (1:2000, Abmart, Shanghai, China), Flotillin-1 (1:1000, CST) and GAPDH (1:5000, Abmart, Shanghai, China) antibodies by previously described methodology[8].

\section{Cell culture}

The HUVECs were isolated from 3 individual donors by a standard collagenase enzyme digestion method and cultured steadily in Endothelial Cell Medium (ECM, ScienCell, San Diego, CA) containing 5\% FBS, 1\% $\mathrm{P} / \mathrm{S}$ and $1 \%$ ECGS.

\section{Tet-One induced sFlt-1 expression in HUVECs}

The human sFIt-1 (ID: NM_001159920.1) cDNA was cloned into Lenti-X Tet-One System expression vector (Clontech, Moutain View, CA) as described previously. The recombinant lentivirus tet-sFlt-1 and the negative control lentivirus (NC-lentivirus; Hanyin Co. Shanghai, China) were prepared and titered to $10^{9}$ $\mathrm{TU} / \mathrm{ml}$ (transfection unit). HUVECs were infected with lentiviruses expressing Tet-on-sFlt-1 to obtain cells overexpressing sFlt-1 only at the time when tetracycline existing. HUVECs expressed the sFlt-1 in the cellular background with doxycycline (Dox) and the efficiency of overexpression was examined by western blot analysis.

\section{Exosome labelling}

Exosomes were labeled with the fluorescent dye 1,10-dioctadecyl-3,3,30,30-tetramethylindocarbocyanine perchlorate (Dil, red, Sigma) by addition to PBS and incubated for 15 min according to the manufacturer's protocol [14]. The labeled exosome suspensions were filtered using a 100-kDa MWCO hollow fiber membrane (Thermo Fisher Scientific) to remove the excess dye. HUVECs were seeded in 6-well plates and incubated with the Dil-labeled exosomes $(100 \mu \mathrm{g} / \mathrm{mL})$ for $24 \mathrm{~h}$. Then the cells were fixed with $4 \%$ paraformaldehyde for 15 minutes and stained with phalloidin-FITC (Sigma). Nuclei were stained with 4',6- 
diamidino-2-phenylindole (DAPI, Sigma). The cellular uptake of exosomes was tested by a confocal microscopy (TCS SP8; Leica, Wetzlar, Germany).

\section{Cell proliferation and migration assays}

Cell proliferation and migration ability was performed using modified systems as described previously[15]. In brief, $3 \times 10^{3}$ cells/well were plated in 96-well plates, later Dox or the vehicle were added to induce overexpression of sFlt-1. After $24 \mathrm{~h}, \mathrm{HUCMSC}$-exos with different doses were added. Then cell numbers were assessed using the cell counting kit-8 (CCK-8) at $450 \mathrm{~nm}$. For migration, HUVECs were plated in 6-well plates and treated with Dox or the vehicle to induce overexpression of sFlt-1. Then $3 \times 10^{4}$ cells were seeded into the upper chambers and exosomes $(100 \mu \mathrm{g} / \mathrm{mL})$ or NS were added into the lower chambers. Following incubation for $16 \mathrm{~h}$, fluorescent stain (calcein-AM) was added to each lower chamber. The migrated cells were counted by fluorescence analysis (Nikon, Tokyo, Japan).

\section{Proteomic analysis}

The HUCMSCs-exo and HUCMSCs samples were processed for tandem mass tag (TMT)-based quantitative proteomic analysis by Lu-Ming Biotech Co., Ltd. (Shanghai, China). High-performance liquid chromatography tandem mass spectrometry (HPLC-MS/MS) was used to compare the proteomic content of HUCMSCs-exo and cells. Differentially expressed proteins were identified with a cutoff of absolute fold change $\geq 2$ and $P$-value $\otimes 0.05$

\section{Statistics}

All data were expressed as the mean \pm the standard error of the mean (S.E.M) and analyzed using the SPSS 23.0 statistical analysis software (SPSS Inc., Chicago, IL, USA). Statistical significance was determined by performing paired Student's t-test, one-way analysis of variance (ANOVA) and Dunett's post-hot test. A p-value $<0.05$ was considered statistically significant.

\section{Results}

\section{Isolation and characterization of HUCMSC-exos}

We isolated HUCMSCs from human umbilical cord Wharton's jelly using the tissue adherence method[16]. Observation under an inverted microscope showed that the HUCMSCs (passage 0) had long fusiform shapes (Figure 1A). After osteogenic induction, alizarin red staining showed multiple calcium nodules in the cells, which indicated that the cells had well-developed osteogenic differentiation functions (Figure 1B). Flow cytometric analysis showed that the HUCMSCs were positive for CD73, CD90 and CD105 but lacked the hematopoietic marker CD45 (Figure 1C), which was consistent with the characteristics of MSCs.

The characteristics of HUCMSC-exos isolated and purified using a well-established ultracentrifuge method are presented [17]. Our hypothesis was supported by transmission electron microscopy analysis, 
which showed a cup shape (Figure 1D). NTA identified particles with diameters of 30-150 nm (Figure 1E). Furthermore, exosomes were positive for the exosomal protein markers CD63, CD81 and CD9 (Figure 1F). These results indicated that we successfully isolated HUCMSC-exos.

\section{HUCMSC-exos ameliorated sFlt-1-induced PE-like adverse birth outcomes in pregnant mice}

We first established a widely used PE-like mouse model by injecting $6 \times 10^{8}$ pfu of the recombinant sFlt-1expressing adenovirus into pregnant ICR mice via the tail vein on E8.5. In accordance with our previous findings, exosomes from human fluids can enter murine organs[8], thus, HUCMSC-exos were administered to examine the potential of exosomes in treating PE. Pregnant mice were injected with exosomes or diluent on E6.5, E9.5, E12.5 and E15.5 (Figure 2A). Hypertension is the most common diagnostic sign of $\mathrm{PE}$, then blood pressure response in the sFlt-1-induced preeclampsia model was examined and showed in Figure 2C. The sFlt-1-injected mice had both increased systolic and diastolic blood pressure. No significant differences were observed among the CTL and EXO groups, while dams treated with both sFlt-1 and HUCMSC-exos remained nearly normotensive on E18.5 relative to that of dams treated only with sFlt-1. SFIt-1 injection induced the production of sFlt-1 and increased its serum concentration in mice (Figure 2E, sFlt-1 vs CTL, P< 0.01) without affecting sEndoglin (sEng) levels. Circulating sFlt-1 concentrations were slightly decreased by exosome treatment (sFlt-1 vs EXO + sFlt-1, P $=0.13)$.

The effects of exosomes on PE-induced IUGR were evaluated, and the observed decrease in body weight (Figure 2B) was most likely caused by small-sized fetuses and placentas rather than a reduction in the number of fetuses (Figure 2F). Fetuses that survived to term had decreased weights and were small in the sFlt-1-treated group, and exosomes prevented this growth restriction in fetuses and placentas (Figure 2F). The urine albumin/creatinine ratio (ACR) was slightly increased after sFlt-1 injection, but the difference was not significant (Figure 2D, $P=0.19$ ). These results suggest that HUCMSC-exos significantly reduce the IUGR that occurs in the fetuses of dams exposed to sFIt-1. Therefore, HUCMSC-exos have the potential to treat PE by improving sFlt-1-induced hypertension and IUGR in mice.

\section{HUCMSC-exos improved placental vascular development in PE-like mice}

The sFlt-1 virus-treated mice showed retarded fetal development, which is driven by impaired placental feto-maternal exchange. After exchange of gases and nutrients between the maternal and fetal circulation in the maze of labyrinth (La), deoxygenated blood then moves to the junctional zone (JZ) [18]. Thus, we examined the histology of the placentas, including the La and JZ by H\&E and IHC staining (Figure 3A). The total placental area did not change among the four groups (Figure 3B), while the thickness of the La layer was notably reduced in sFlt-1-injected mice and rescued by HUCMSC-exo (Figure 3C-D). Then, we analyzed placental vascularization by staining for CD31, which specifically binds to endothelial cells. High magnification views showed extensive vascular damage in the La, which indicated impaired blood flow, in the sFlt-1 group. Importantly, in EXO+sFlt-1 mice, there was a partial reversal of the vascular narrowing observed in placentas from sFlt-1 mice, as indicated by increased lumen diameters 
and areas (Figure 3E). The number of lumens per image in the exosome-treated group relative to the sFlt1 group was slightly increased but was not significant (Figure 3F). Quantification of vascular diameter after CD31 staining showed that this decreased from $22.1 \pm 1.95 \mu \mathrm{m}$ in CTL mice to $14.45 \pm 1.64 \mu \mathrm{m}$ in sFlt-1 mice $(P<0.01)$ and reversed to $21.6 \pm 1.44 \mu \mathrm{m}$ in EXO+sFlt-1 mice $(P<0.01)$ (Figure $3 \mathrm{G})$. Furthermore, the fetal vascular area (\%) was reduced in sFlt-1 mice (19.49 \pm 0.71 vs $25.23 \pm 1.38, p<$ $0.001)$ and increased to $22.75 \pm 1.4$ in EXO+sFlt-1-treated mice $(p=0.06)$ (Figure $3 H)$. There was no significant difference in the vascular number, diameter, or area between EXO and CTL mice. Neither glomerular endotheliosis nor mesangial expansion, which are characteristic features of $P E[19]$, was present in any group, as determined by silver staining (Figure 3I).

\section{In vitro proangiogenic effects of HUCMSC-exos}

A substantial body of evidence indicates that PE in humans is a consequence of vascular endothelial damage, resulting in multiorgan dysfunction. Therefore, we chose HUVECs as the cell model for studying the effects of HUCMSC-exos on endothelial dysfunction in PE. The Tet-On-sFlt- Flag $_{\text {Fla }}$ HUVC model was established to simulate vascular endothelial cells in women with PE. In the absence of Dox, negative control (NC) HUVECs did not express any detectable sFlt-1 protein, and following induction with different doses of Dox, strong signals for sFlt-1 were observed in sFlt-1-overexpressing (OV-sFlt-1) cells, as measured by western blotting (Figure 4A). HUCMSC-exos labeled with the fluorescent dye Dil were incubated with NC and OV-sFlt-1 HUVECs for $24 \mathrm{~h}$, and most recipient cells were positive for Dil fluorescence and showed no difference among the groups (Figure 4B).

A series of cellular functional analyses were performed, and we first demonstrated that HUCMSC-exos are capable of facilitating angiogenesis because they contain many proangiogenic components. OV-sFlt-1HUVEC proliferation and migration were significantly inhibited, and these attenuated angiogenetic behaviors were reversed after incubation with HUCMSC-exos (Figure 4C-D). It is suggested that high levels of circulating sFlt-1 lead to suppression of eNOS signaling pathway, which, in turn, enhances sensitivity to vasoconstrictors that induces maternal hypertension in $\mathrm{PE}[20]$. Therefore, we examined the protein expression of eNOS in HUVECs and as expected, found that OV-sFIt-1-HUVECs exhibited decreased eNOS protein expression, which was partially rescued in the presence of HUCMSC-exos (Figure $4 \mathrm{E})$. These findings suggest that HUCMSC-exos have the potential protective effects on sFlt-1-induced endothelial and eNOS dysfunction.

\section{Proteomic analysis of HUMSC and HUCMSC-exos}

HUCMSC-exos modulated sFlt-1-induced endothelial dysfunction and improved placental angiogenesis in PE-like mice. It was therefore decided to investigate how exosomes enhanced vascular angiogenesis. After successfully extracting proteins from exosomes and parallel cells, proteomics analysis using tandem mass tag (TMT) technology was performed to detect protein expression profiles. Unsupervised hierarchical clustering analysis was used to generate a heat map of the differentially expressed proteins, and a total of 568 proteins were differentially expressed between HUCMSC-exos and HUCMSCs using the 
cutoff value of a 2 -fold change and a $\mathrm{P}$ value $<0.05$ (Figure 5A). On the basis of gene ontology (G0) enrichment analysis, both HUCMSC and HUCMSC-exos displayed functional enrichment in biological processes related to vesicle-mediated transport, cell communication, cell migration, and angiogenesis (Figure 5B). Kyoto Encyclopedia of Genes and Genomes (KEGG) pathway enrichment analysis showed the oxidative phosphorylation, focal adhesion, endocytosis, and extracellular matrix-receptor (ECMreceptor) were enriched pathways (Figure $5 \mathrm{C}$ ). We next analyzed the differentially expressed genes (DEGs) between these two chambers. Compared with HUCMSCs, the up-expressed proteins in HUCMSCexos were MMP2 (Matrix metalloproteinase 2, fold change $=14.7$ ) and VCAN (versican, fold change = 11.7). Previous study showed that MSC-exo could promote the migration of endothelium cells by delivering MMP2 [21]. VCAN contributes to tissue development and maintenance by participating in cell adhesion, proliferation and angiogenesis via binding to ECM components [22]. Then, we verified that VCAN was obviously upregulated in HUCMSC-exos compared with cell lysates (Data were not shown).

\section{Discussion}

$\mathrm{PE}$ is a unique multisystem disorder that leads to maternal and fetal morbidity and mortality. Therapeutic strategies aimed at reducing blood pressure and improving fetal birth outcomes are being pursued as avenues to prevent $\mathrm{PE}[23]$. In the current study, we showed that HUCMSC-exos provided therapeutic benefits in animal models of PE by improving birth outcomes and vascular endothelium functions.

Compelling evidence indicates that MSCs and their derived exosomes can be used in many vascular dysfunction-related diseases. Due in part to studying the paracrine effects of stem cell therapy, this specific therapy has been mechanistically linked to the inherited functions of secreting exosomes[24]. Recently, research suggested HUCMSC-originated exosomes might be an innovative direction for therapeutic approaches against PE by accelerating trophoblast cell invasion and migration[21, 25]. Despite the therapeutic effects observed, however, the underlying molecular mechanism, especially how the vascular is regulated, remains unclear. In the current study, we focused on HUCMSC-exos as potential cell-free therapeutics for the treatment of PE by improving angiogenesis. We chose a well-established mouse model of PE that mimics many features of the human pregnancy disease, including upregulated blood pressure and serum sFlt-1 concentration, and is established by a single injection of sFlt-1 adenovirus[20]. Administration of HUCMSC-exos during pregnancy prevented the development of sFlt-1induced preeclamptic complications, decreasing blood pressure and improving fetal and placental weights.

Modified vascular responses are prevented in hypertensive pregnancy complications due to an imbalance in angiogenetic and antiangiogenetic factors. Circulating sFlt-1 levels are considered a diagnostic and prognostic marker of $\mathrm{PE}$, and sFlt-1 is a clinically promising therapeutic target for this disease. In our mouse model, administration of HUCMSC-exos slightly decreased the serum sFlt-1 concentration. Insufficient placental vascularization and reduced blood flow to the feto-placental unit resulted in impaired fetal growth in PE. Placental vascular network density in the labyrinth zone of sFlt-1-treated mice was sparse, and the fetal sinusoids were narrow. The mice treated with HUCMSC-exos had improved 
fetal and placental weight and size as the result of a decrease in the diffusion distance between the fetal and maternal blood supplies that provided enough nutrition for the developing fetus. Widespread vascular damage is an invariable finding in $\mathrm{PE}[26]$, and we indicated the addition of HUCMSC-exos recused cell proliferation and migration abilities of OV-sFlt-1-HUVECs in vitro. NO-mediated reductions in blood pressure are crucial, and decreased NO bioavailability can lead to hypertension by enhancing vascular oxidative stress and endothelial dysfunction. OV-sFIt-1-mediated interference in eNOS expression in HUVECs could be reversed by exosomes. Thus, HUCMSC-exos may serve as a novel medication that rescues endothelial dysfunction in treating PE.

Exosomes have therapeutic potential owing to their roles as carriers to deliver nucleic acids and proteins between human body systems. In addition, genetic or molecular engineering of exosomes can improve target specificity and anti-disease activity with less toxicity. Based on our proteomic analysis, a better understanding of the molecular and cellular properties of HUCMSC-exos contributes to their therapeutic potential as innovative drug delivery systems. Exosomal MMP2 derived from mature osteoblasts was previously demonstrated to promote endothelial angiogenesis via VEGF/Erk1/2 signaling pathway[27]. VCAN, which highly expressed in metabolically active tissues can mediate angiogenesis possibly depends upon interactions with VEGF and influence the assembly of the ECM[28]. Upon uptake by the vascular endothelium, these proteins accumulated in HUCMSC-exos promoted cell proliferation, migration and angiogenesis to rescue damaged vascular tissues in preeclamptic-like mice.

\section{Conclusion}

In summary, we reported the beneficial effects of exosomes derived from HUCMSCs in sFlt-1-induced preeclamptic mice by promoting angiogenesis. Thus, we provide new evidence for the consideration of HUCMSC-exos as a novel therapeutic approach to PE treatment.

\section{Abbreviations}

\section{HUCMSC}

Human umbilical cord mesenchymal stem cell

\section{sFlt-1}

Soluble fms-like tyrosine kinase-1

\section{HUCMSC-exos}

Human umbilical cord mesenchymal stem cell-derived exosomes

HUVEC

Human umbilical vein endothelial cell

PE

Preeclampsia

\section{IUGR}

Intrauterine growth retardation

\section{MHC-II}


Major histocompatibility complex-II

FBS

Fetal bovine serum

BSA

Bovine serum albumin

DOX

Doxycycline

TEM

Transmission electron microscope

NTA

Nanoparticle tracking analysis

H\&E

Hematoxylin and eosin

PAS

Periodic acid-methenamine silver

NC

Negative control

TMT

Tandem mass tag

ANOVA

Analysis of variance

ACR

Albumin/creatinine ratio

La

Labyrinth

JZ

Junctional zone

MMP2

Matrix metalloproteinase 2

ECM

Extracellular matrix

VCAN

Versican

\section{Declarations}

\section{Ethics approval and consent to participate}

The collection of human umbilical cord tissues was approved by the Research and Ethics Committee of Shanghai First Maternity and Infant Hospital. All mouse experiments were approved by the Department of Laboratory Animal Science, Tongji University. 


\section{Consent for publication}

Not applicable.

\section{Availability of data and materials}

The raw data is available from the authors on reasonable request.

\section{Competing interests}

The authors declare that they have no competing interests.

\section{Funding}

This work was supported by the National Natural Science Foundation of China (No. 82001567 and 81771591) and sponsored by Shanghai Sailing Program (No. 20YF1438400).

\section{Authors' contributions}

Xinwen Chang was a major contributor in conducting the experiments and writing the manuscript. Qizhi He performed the histological examination of the tissues and analyzed the data. Mingmin Wang established the mouse experiments. Linyan Jia collected the human umbilical cord tissues. Tao Duan provided funding and revised the manuscript. Kai Wang designed the experiment and revised the manuscript. All authors read and approved the final manuscript.

\section{Acknowledgements}

Not applicable.

\section{References}

1. Redman CW, Sargent IL. Latest advances in understanding preeclampsia. Science (New York, NY). 2005;308(5728):1592-4. Epub 2005/06/11. doi: 10.1126/science.1111726. PubMed PMID: 15947178.

2. Souza JP, Gulmezoglu AM, Vogel J, Carroli G, Lumbiganon P, Qureshi Z, et al. Moving beyond essential interventions for reduction of maternal mortality (the WHO Multicountry Survey on Maternal and Newborn Health): a cross-sectional study. Lancet. 2013;381(9879):1747-55. doi:10.1016/s0140-6736(13)60686-8. PubMed PMID: 23683641. Epub 2013/05/21.

3. Stepan H, Geide A, Faber R. Soluble fms-like tyrosine kinase 1. The New England journal of medicine. 2004;351(21):2241-2. Epub 2004/11/19. doi: 10.1056/nejm200411183512123. PubMed PMID: 15548791.

4. Yang W, Zhang J, Xu B, He Y, Liu W, Li J, et al. HucMSC-Derived Exosomes Mitigate the Age-Related Retardation of Fertility in Female Mice. Mol Ther. 2020;28(4):1200-13. 
doi:10.1016/j.ymthe.2020.02.003. PubMed PMID: 32097602; PubMed Central PMCID: PMCPMC7132622. Epub 2020/02/26.

5. Bier A, Berenstein P, Kronfeld N, Morgoulis D, Ziv-Av A, Goldstein H, et al. Placenta-derived mesenchymal stromal cells and their exosomes exert therapeutic effects in Duchenne muscular dystrophy. Biomaterials. 2018;174:67-78. doi:10.1016/j.biomaterials.2018.04.055. PubMed PMID: 29783118. Epub 2018/05/22.

6. Huang Y, Wu Y, Chang X, Li Y, Wang K, Duan T. Effects of Human Umbilical Cord Mesenchymal Stem Cells on Human Trophoblast Cell Functions In Vitro. Stem cells international. 2016;2016:9156731. doi:10.1155/2016/9156731.

7. Ruivo CF, Sugimoto H, Lee JJ, Kalluri R, Melo SA, Yang S, et al. Exosomes facilitate therapeutic targeting of oncogenic KRAS in pancreatic cancer. Nature. 2017;546(7659):498-503.

8. Chang X, Yao J, He Q, Liu M, Duan T, Wang K. Exosomes From Women With Preeclampsia Induced Vascular Dysfunction by Delivering sFlt (Soluble Fms-Like Tyrosine Kinase)-1 and sEng (Soluble Endoglin) to Endothelial Cells. Hypertension (Dallas, Tex: 1979). 2018;72(6):1381-90. Epub 2018/12/21. doi: 10.1161/hypertensionaha.118.11706. PubMed PMID: 30571229.

9. Lei Q, Gao F, Liu T, Ren W, Chen L, Cao Y, et al. Extracellular vesicles deposit PCNA to rejuvenate aged bone marrow-derived mesenchymal stem cells and slow age-related degeneration. Sci Transl Med. 2021;13(578). Epub 2021/01/29. doi:10.1126/scitranslmed.aaz8697. PubMed PMID: 33504653.

10. Eirin A, Zhu XY, Jonnada S, Lerman A, van Wijnen AJ, Lerman LO. Mesenchymal Stem Cell-Derived Extracellular Vesicles Improve the Renal Microvasculature in Metabolic Renovascular Disease in Swine. Cell Transplant. 2018;27(7):1080-95. doi:10.1177/0963689718780942. PubMed PMID: 29954220; PubMed Central PMCID: PMCPMC6158551. Epub 2018/06/30.

11. Li X, Liu LL, Yao JL, Wang K, Ai H. Human Umbilical Cord Mesenchymal Stem Cell-Derived Extracellular Vesicles Inhibit Endometrial Cancer Cell Proliferation and Migration through Delivery of Exogenous miR-302a. Stem cells international. 2019;2019:8108576. Epub 2019/04/20. doi: 10.1155/2019/8108576. PubMed PMID: 31001342; PubMed Central PMCID: PMCPMC6437733.

12. Chang X, Bian Y, He Q, Yao J, Zhu J, Wu J, et al. Suppression of STAT3 Signaling by Delta9Tetrahydrocannabinol (THC) Induces Trophoblast Dysfunction. Cellular physiology and biochemistry: international journal of experimental cellular physiology, biochemistry, and pharmacology. 2017;42(2):537-50. Epub 2017/06/05. doi: 10.1159/000477603. PubMed PMID: 28578322.

13. Li Y, Zhao YJ, Zou QY, Zhang K, Wu YM, Zhou C, et al. Preeclampsia does not alter vascular growth and expression of CD31 and vascular endothelial cadherin in human placentas. The journal of histochemistry cytochemistry: official journal of the Histochemistry Society. 2015;63(1):22-31. Epub 2014/11/02. doi: 10.1369/0022155414558063. PubMed PMID: 25362142; PubMed Central PMCID: PMCPMC4395995.

14. Zhou W, Fong MY, Min Y, Somlo G, Liu L, Palomares MR, et al. Cancer-secreted miR-105 destroys vascular endothelial barriers to promote metastasis. Cancer cell. 2014;25(4):501-15. 
doi:10.1016/j.ccr.2014.03.007. PubMed PMID: 24735924; PubMed Central PMCID:

PMCPMC4016197. Epub 2014/04/17.

15. Chang X, Li H, Li Y, He Q, Yao J, Duan T, et al. RhoA/MLC signaling pathway is involved in Delta(9)tetrahydrocannabinol-impaired placental angiogenesis. Toxicology letters. 2018;285:148 - 55. Epub 2018/01/09. doi:10.1016/j.toxlet.2017.12.031. PubMed PMID: 29307655.

16. Zhilai Z, Biling M, Sujun Q, Chao D, Benchao S, Shuai H, et al. Preconditioning in lowered oxygen enhances the therapeutic potential of human umbilical mesenchymal stem cells in a rat model of spinal cord injury. Brain research. 2016;1642:426 - 35. Epub 2016/04/18. doi:10.1016/j.brainres.2016.04.025. PubMed PMID: 27085204.

17. Goetzl EJ, Schwartz JB, Mustapic M, Lobach IV, Daneman R, Abner EL, et al. Altered cargo proteins of human plasma endothelial cell-derived exosomes in atherosclerotic cerebrovascular disease. FASEB journal: official publication of the Federation of American Societies for Experimental Biology. 2017;31(8):3689-94. Epub 2017/05/10. doi:10.1096/fj.201700149. PubMed PMID: 28476896; PubMed Central PMCID: PMCPMC5503715.

18. Rai A, Cross JC. Development of the hemochorial maternal vascular spaces in the placenta through endothelial and vasculogenic mimicry. Dev Biol. 2014;387(2):131-41. doi:10.1016/j.ydbio.2014.01.015. PubMed PMID: 24485853. Epub 2014/02/04.

19. Li F, Fushima T, Oyanagi G, Townley-Tilson HW, Sato E, Nakada H, et al. Nicotinamide benefits both mothers and pups in two contrasting mouse models of preeclampsia. Proc Natl Acad Sci U S A. 2016;113(47):13450.

20. Burke SD, Zsengeller ZK, Khankin EV, Lo AS, Rajakumar A, DuPont JJ, et al. Soluble fms-like tyrosine kinase 1 promotes angiotensin II sensitivity in preeclampsia. J Clin Investig. 2016;126(7):2561-74. doi:10.1172/jci83918. PubMed PMID: 27270170; PubMed Central PMCID: PMCPMC4922717. Epub 2016/06/09.

21. Liu H, Wang F, Zhang Y, Xing Y, Wang Q. Exosomal microRNA-139-5p from mesenchymal stem cells accelerates trophoblast cell invasion and migration by motivation of the ERK/MMP-2 pathway via downregulation of protein tyrosine phosphatase. J Obstet Gynaecol Res. 2020;46(12):2561-72. doi:10.1111/jog.14495. PubMed PMID: 32945060; PubMed Central PMCID: PMCPMC7756315. Epub 2020/09/19.

22. Nandadasa S, O'Donnell A, Murao A, Yamaguchi Y, Midura RJ, Olson L, et al. The versicanhyaluronan complex provides an essential extracellular matrix niche for Flk1(+) hematoendothelial progenitors. Matrix Biol. 2021. Epub 2021/01/18. doi:10.1016/j.matbio.2021.01.002. PubMed PMID: 33454424.

23. Turanov AA, Lo A, Hassler MR, Makris A, Ashar-Patel A, Alterman JF, et al. RNAi modulation of placental sFLT1 for the treatment of preeclampsia. Nature biotechnology. 2018. Epub 2018/11/20. doi:10.1038/nbt.4297. PubMed PMID: 30451990.

24. Colao IL, Corteling R, Bracewell D, Wall I. Manufacturing Exosomes: A Promising Therapeutic Platform. Trends Mol Med. 2018;24(3):242-56. doi:10.1016/j.molmed.2018.01.006. PubMed PMID: 
29449149. Epub 2018/02/17.

25. Chu Y, Chen W, Peng W, Liu Y, Xu L, Zuo J, et al. Amnion-Derived Mesenchymal Stem Cell ExosomesMediated Autophagy Promotes the Survival of Trophoblasts Under Hypoxia Through mTOR Pathway by the Downregulation of EZH2. Front Cell Dev Biol. 2020;8:545852. doi: 10.3389/fcell.2020.545852. PubMed PMID: 33304896; PubMed Central PMCID: PMCPMC7693549.

26. Nezu M, Souma T, Yu L, Sekine H, Takahashi N, Wei AZ, et al. Nrf2 inactivation enhances placental angiogenesis in a preeclampsia mouse model and improves maternal and fetal outcomes. Science Signaling. 2017;10(479):eaam5711.

27. Tang $\mathrm{H}, \mathrm{He} \mathrm{Y}$, Li L, Mao W, Chen X, Ni H, et al. Exosomal MMP2 derived from mature osteoblasts promotes angiogenesis of endothelial cells via VEGF/Erk1/2 signaling pathway. Exp Cell Res. 2019;383(2):111541. doi:10.1016/j.yexcr.2019.111541. PubMed PMID: 31369752. Epub 2019/08/02.

28. Zheng PS, Wen J, Ang LC, Sheng W, Viloria-Petit A, Wang Y, et al. Versican/PG-M G3 domain promotes tumor growth and angiogenesis. FASEB J. 2004;18(6):754-6. Epub 2004/02/10. doi: 10.1096/fj.03-0545fje. PubMed PMID: 14766798.

\section{Figures}


A

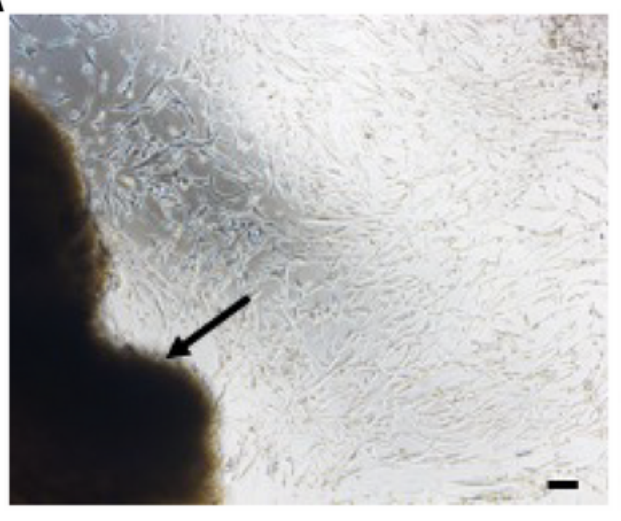

B

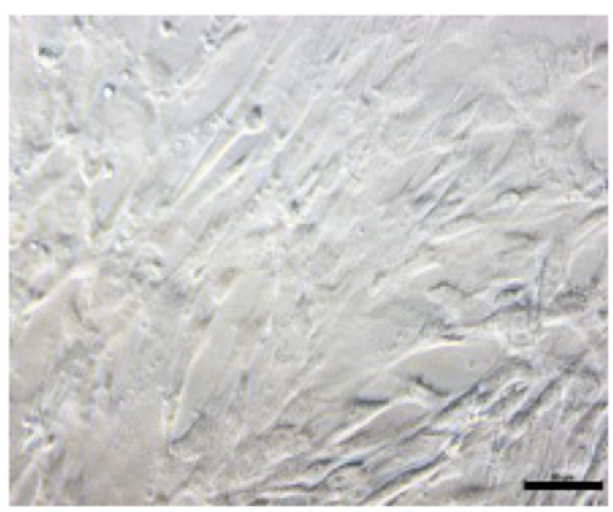

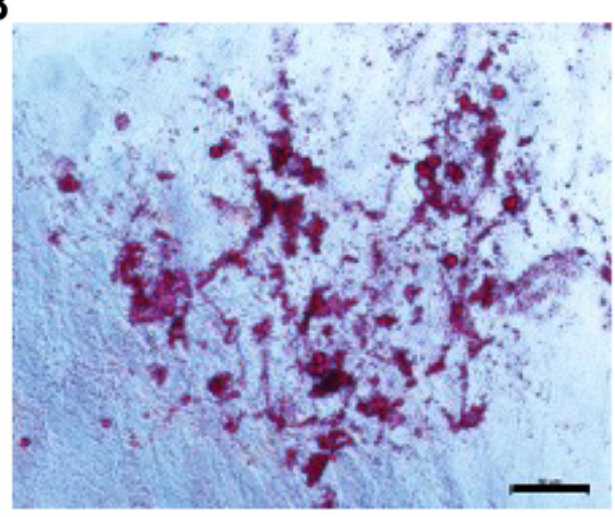

C

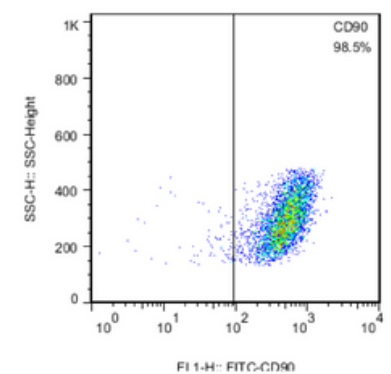

D

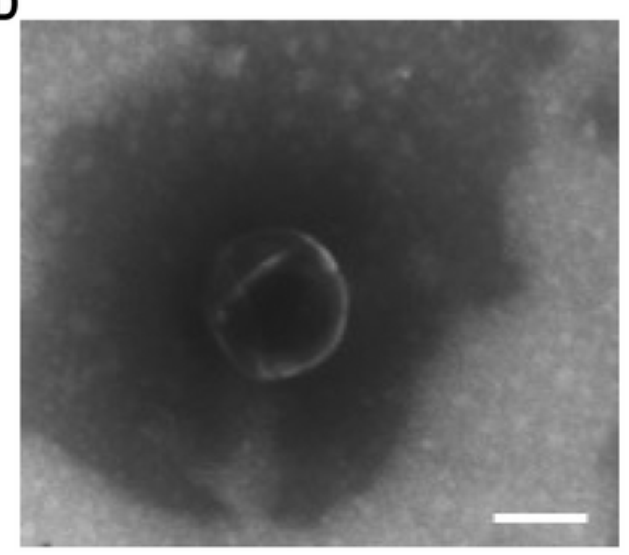

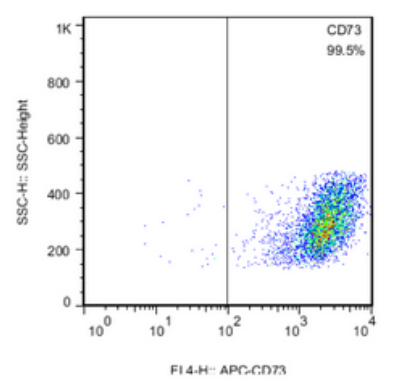

E

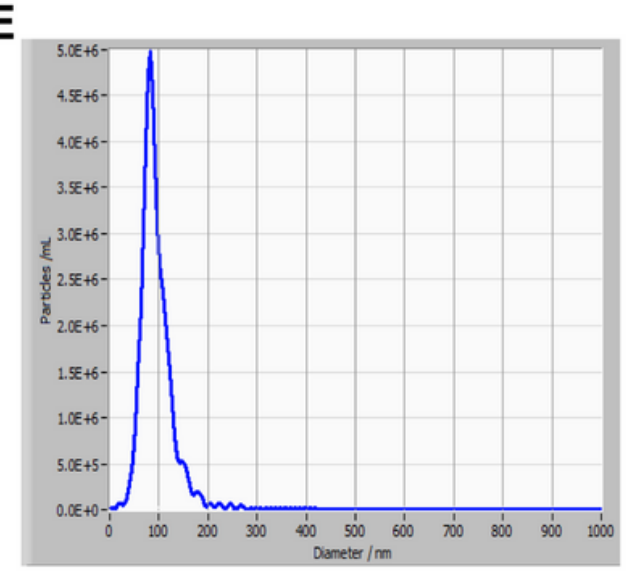

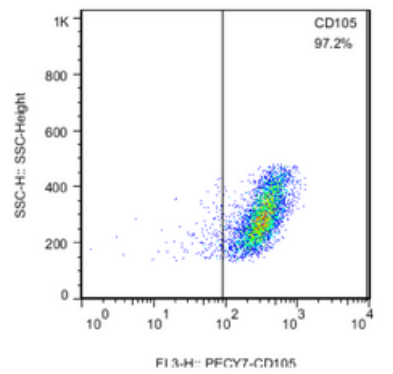

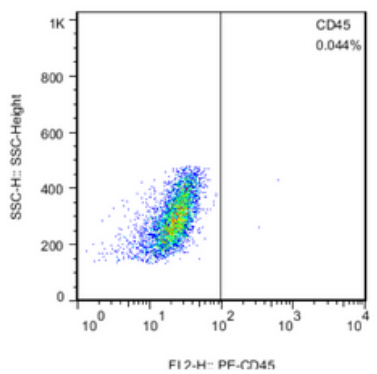

$\mathbf{F}$

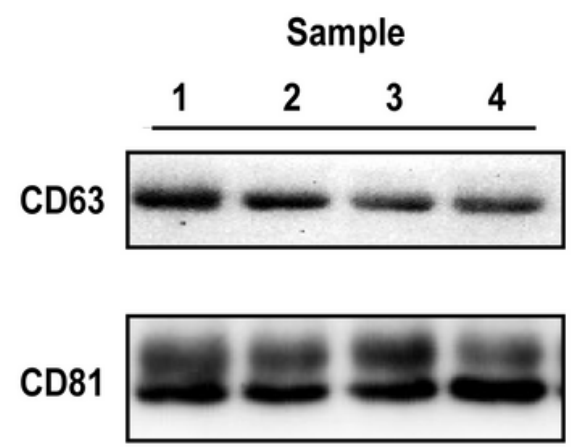

\section{Figure 1}

Identification of HUCMSCs and their derived exosomes. a cell morphology under an inverted microscope, scale bar, arrows indicated the tissue section. b Osteogenic differentiation of hUCMSCs stained positive with alizarin red. Scale bar $100 \mu \mathrm{m}$. c Identification of HUCMSCs by flow cytometry for CD90, CD73, CD105 (positive markers), and CD45 (negative marker). d-f Exosomes collected from HUCMSCs supernatant by ultracentrifuge. Electron micrograph of exosomes, scale bar 200nm (d). Representative vesicle size (nm) distribution by NTA (e). Western blot analysis for the CD63, CD81 and CD9 exosomes enriched marker of HUCMSCs-exo from 4 individual prengnant women (f). 
(i) CTL (control): NS $(100 \mu \mathrm{L})$

(ii) EXO: hUCMSC- exosomes $(100 \mu \mathrm{g})$

(iii) sFlt-1: NS $(100 \mu \mathrm{L})$

(iv) sFIt-1+EXO: hUCMSC- exosomes $(100 \mu \mathrm{g})$

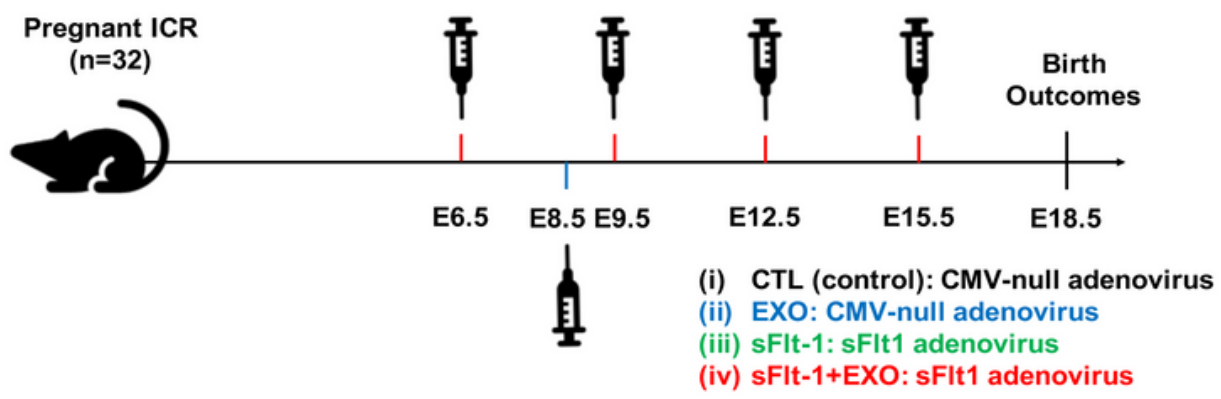

B

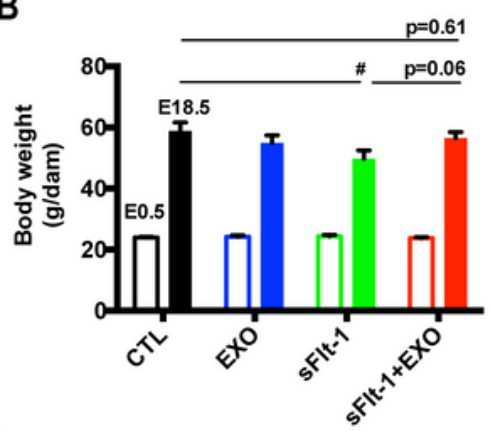

D

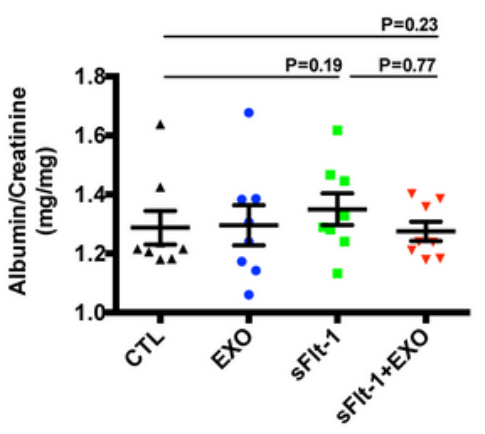

$\mathbf{F}$

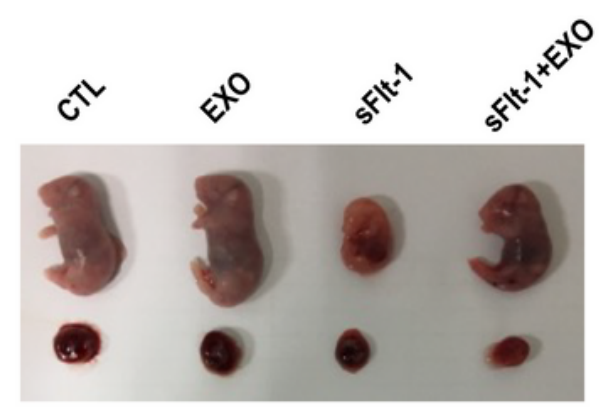

C

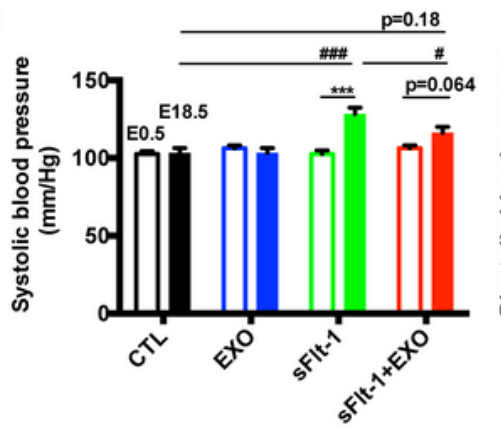

E

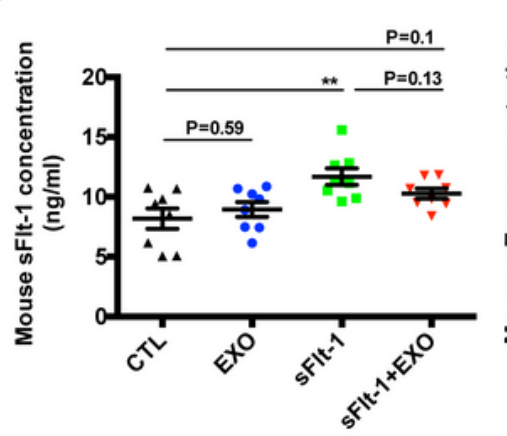

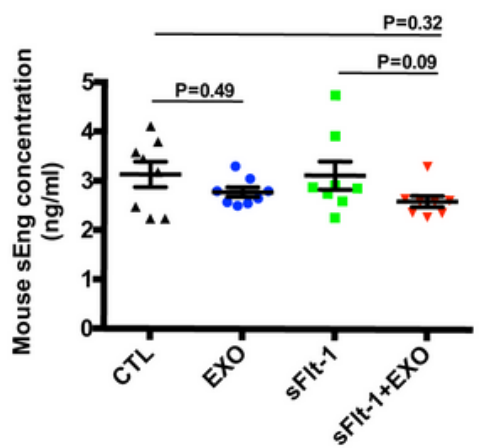
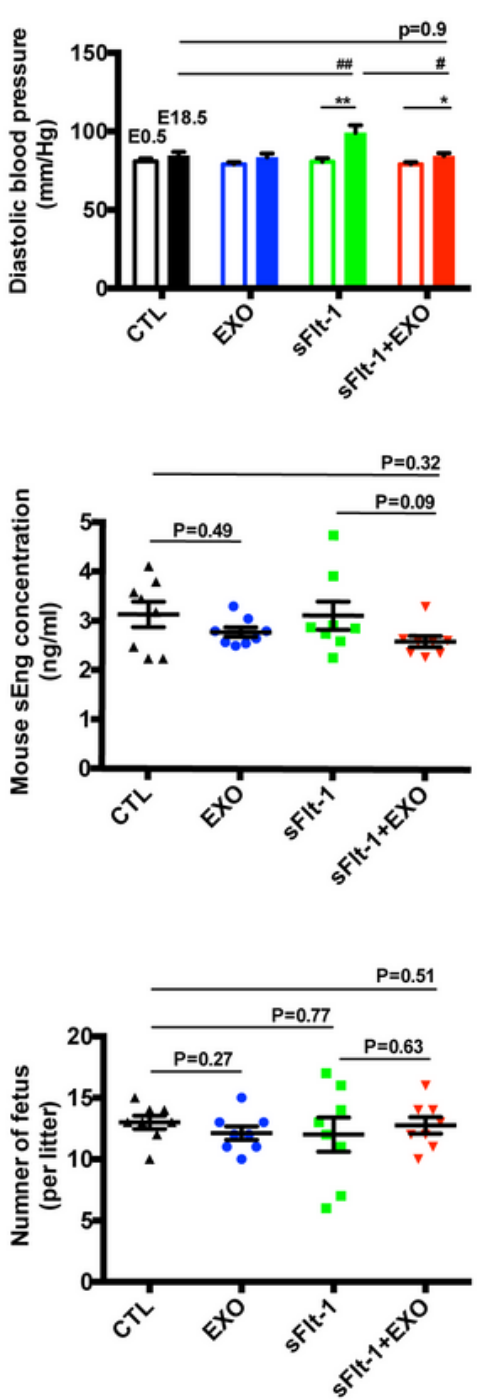

Figure 2

HUCMSCs-exosomes improve the sFlt-1-induced preeclamptic mouse reproductive outcomes. a Pregnant mice were randomly divided into four groups and respectively treated with nature saline (i, CTL, n=8), HUCMSCs-exosomes (ii, EXO, $100 \mu \mathrm{g}, \mathrm{n=8}$ ), nature saline (iii, sFlt-1, n=8) or HUCMSCs-exosomes (iv, sFlt-

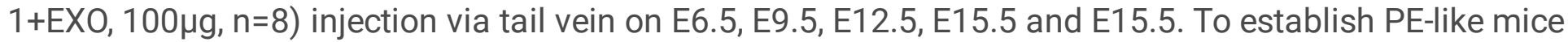
model, pregnant mice were injected with CMV-null adenovirus (i, CTL; ii, EXO) amd sFlt-1 adenovirus (iii, 
sFlt-1; iv, sFlt-1+EXO) on E8.5. b Body weights of dams on E0.5 and E18.5 (one-way ANOVA and Dunett's post-hot tests, sFlt-1 vs CTL on E18.5, \#p<0.05). c Blood pressure measurements on E0.5 and E18.5 (Paired students' t-tests, E18.5 vs E0.5, ${ }^{\star} \mathrm{p}<0.05,{ }^{* \star} \mathrm{p}<0.01$, ${ }^{* \star *} \mathrm{p}<0.001$; Systolic blood pressure on E18.5, one-way ANOVA and Dunett's post-hot tests, sFlt-1 vs CTL, \#\#\#p<0.001, sFlt-1+EXO vs sFlt-1, \#p<0.05; Diastolic blood pressure on E18.5, sFlt-1 vs CTL, \#\#p<0.01, sFlt-1+EXO vs sFlt-1). d Ratio of albumin/creatinine in mice urine was measured by ELISA kit on E18.5. e Mouse sFlt-1 and sEng concentrations in serum were measured by ELISA kits on E18.5 (one-way ANOVA and Dunett's post-hot tests, sFlt-1 vs CTL, \#\#p<0.01). f Effects of HUCMSC-exos-injection on morphology, birth weights and number of fetuses per litter. ( $\mathrm{n}=8$ per group of dams, one-way ANOVA and Dunett's post-hot tests, sFlt- 1 vs CTL, \#\#\#p<0.001, sFlt-1+EXO vs sFlt-1, \#p<0.05). 


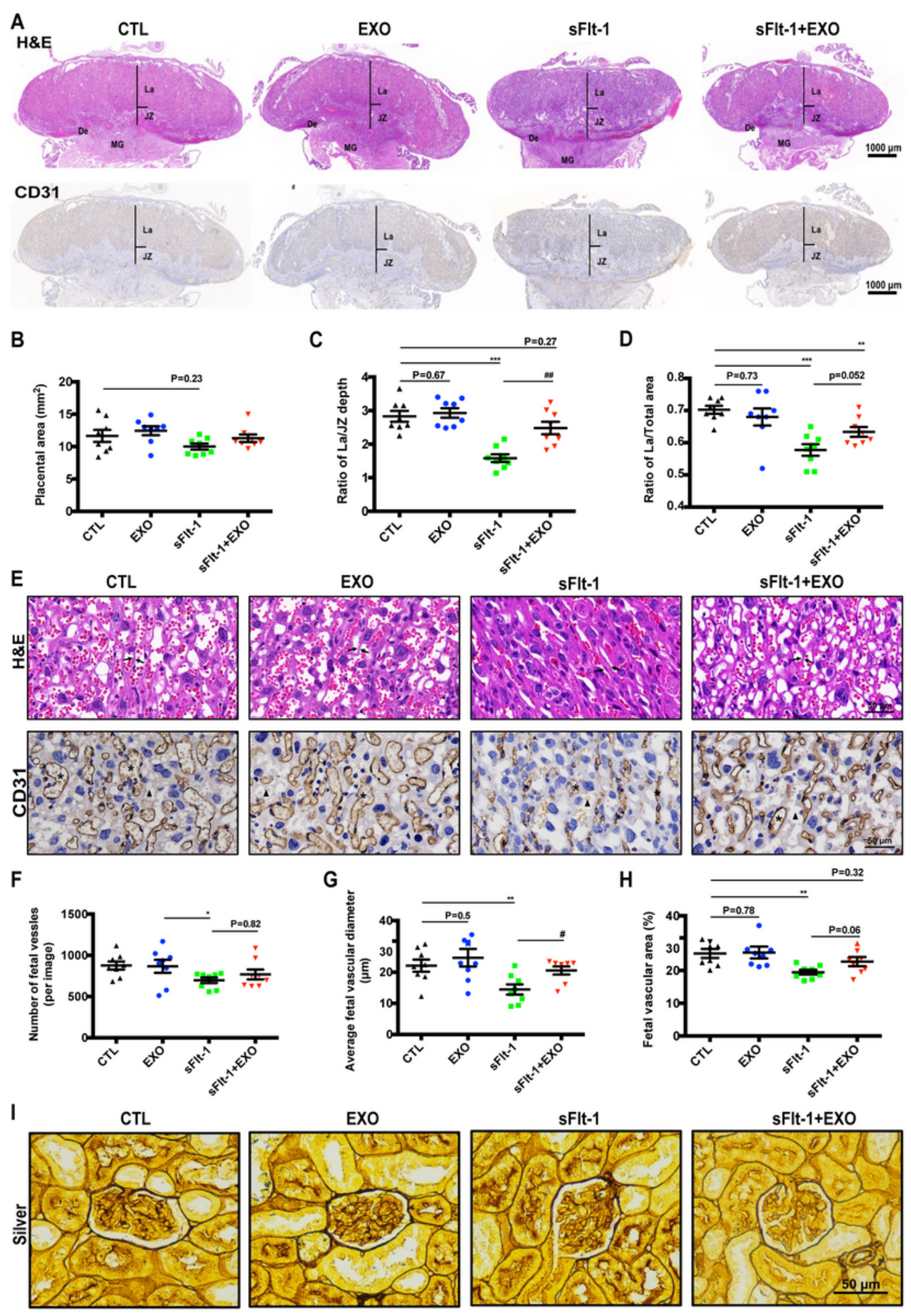

\section{Figure 3}

Effects of HUCMSC-exos on histopathology of mice placenta and kidney. a H\&E staining and IHC staining for CD31 of mice placentas (JZ, jountional zone; L, labyrinth), scale bar $2000 \mu \mathrm{m}$. b Placental area of pregnant mice. c Ratio of La/JZ depth of placenta. d Ratio of La/total area of placenta. e H\&E and IHC staining for CD31 in mice placental tissues. Brown color indicates positive staining for CD31. Arrowheads: trophoblastic septa; asterisk: fetal vasclar; triangle: intervillous space. scale bar 50 $\mu \mathrm{m}$. 
Numbers of fetal vessles (f), average fetal vascular diameter (g) and fetal vascular area (h) in JZ were measured ( $n=8$ per group of dams, one-way ANOVA and Dunett's post-hot tests, * sFlt-1 vs CTL, * $p<0.05$, ${ }^{* *} \mathrm{p}<0.01$, \# sFlt-1-exo vs sFlt-1, \#p<0.05). i Staining of kidney sections with PAS, scale bar $50 \mu \mathrm{m}$.

A

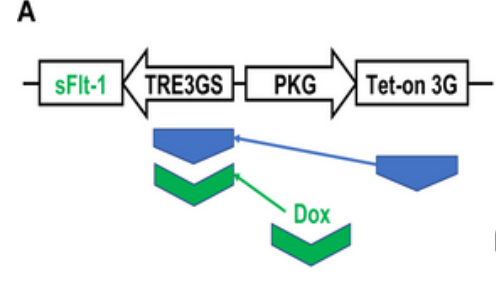

B

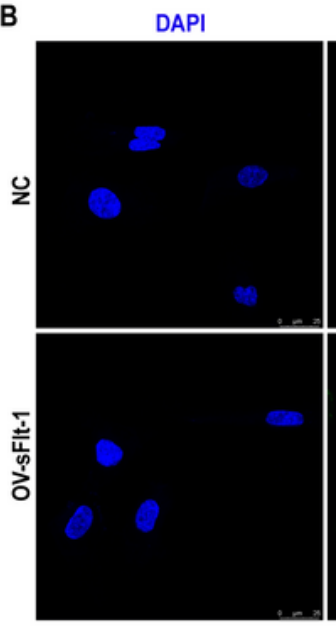

C
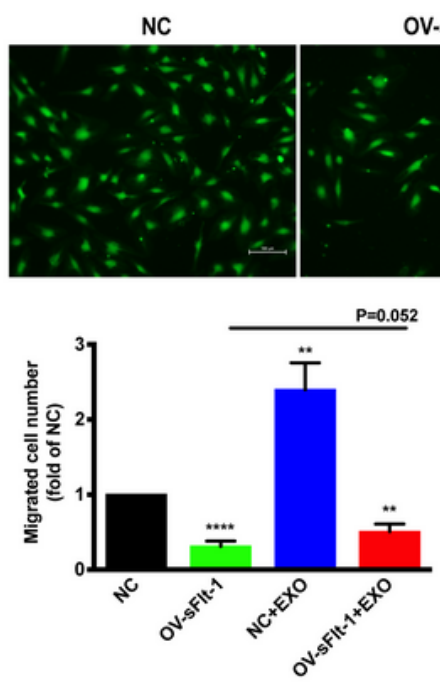

E

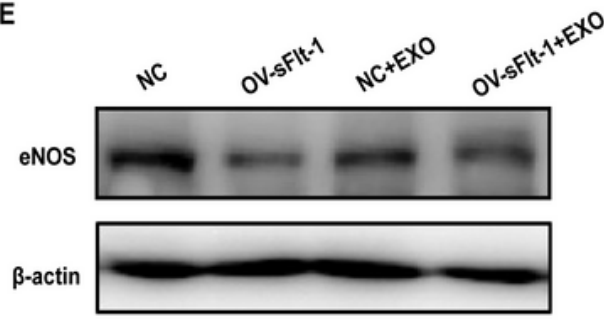

$\beta$-actin

OV-sFIt-1
Dox (ng/ml)

$\begin{array}{lllll}0 & 25 & 50 & 100 & 200\end{array}$
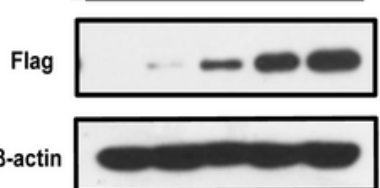

Dil-labeled
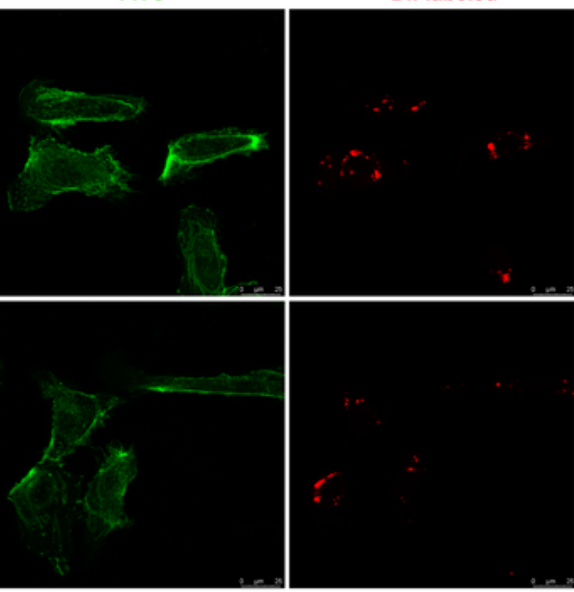

NC+EXO

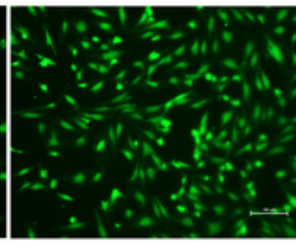

D
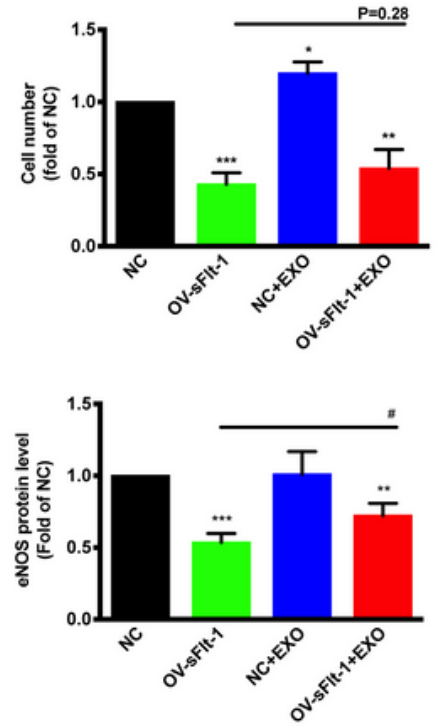

Figure 4

Proangiogenic effects of HUCMSC-exos. a Stably transfected HUVECs were treated with the indicated concentrations of Dox for 24h. Western blot analysis of sFlt-1 in HUVECs was performed using anti-Flag 
and anti-sFlt-1 antibody. b NC-HUVECs and OV-sFlt-1-HUVECs were incubated with dil-labeled HUCMSCexos (red) for $24 \mathrm{~h}$ before fluorescent and phase contrast images were captured, scale bar $20 \mu \mathrm{m}$. c Cell migration response to HUCMSC-exos $(100 \mu \mathrm{g} / \mathrm{mL})$ was determined by transwell assay, scale bar $100 \mu \mathrm{m}$. d After incubation with HUCMSC-exos $(100 \mu \mathrm{g} / \mathrm{mL})$, HUVEC proliferation was analyzed by CCK-8 assay. e Protein expression of eNOS in HUVECs were detected by western blot. All data were means \pm S.E.M. of 3 pairs of independent experiments performed. One-way ANOVA, ${ }^{*} p<0.05,{ }^{*} p<0.01,{ }^{* *} p<0.001$

A
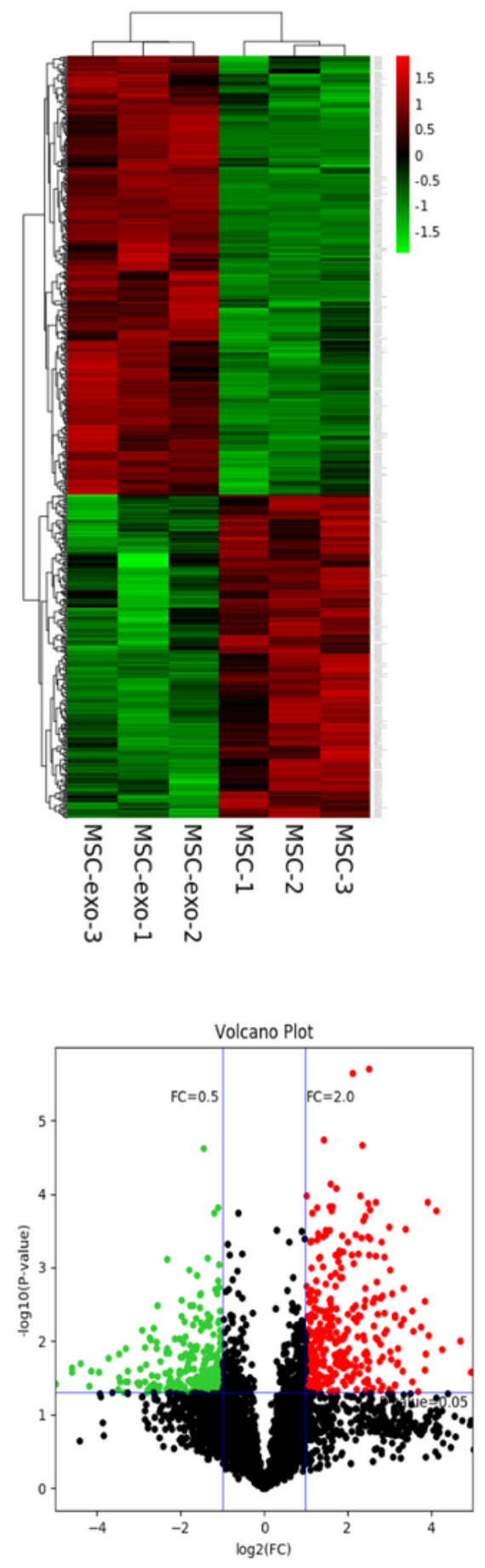

B

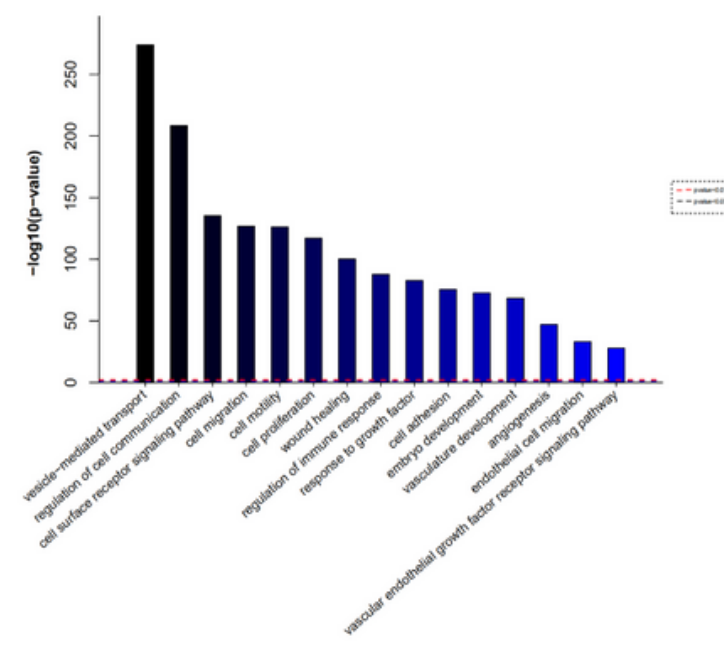

C

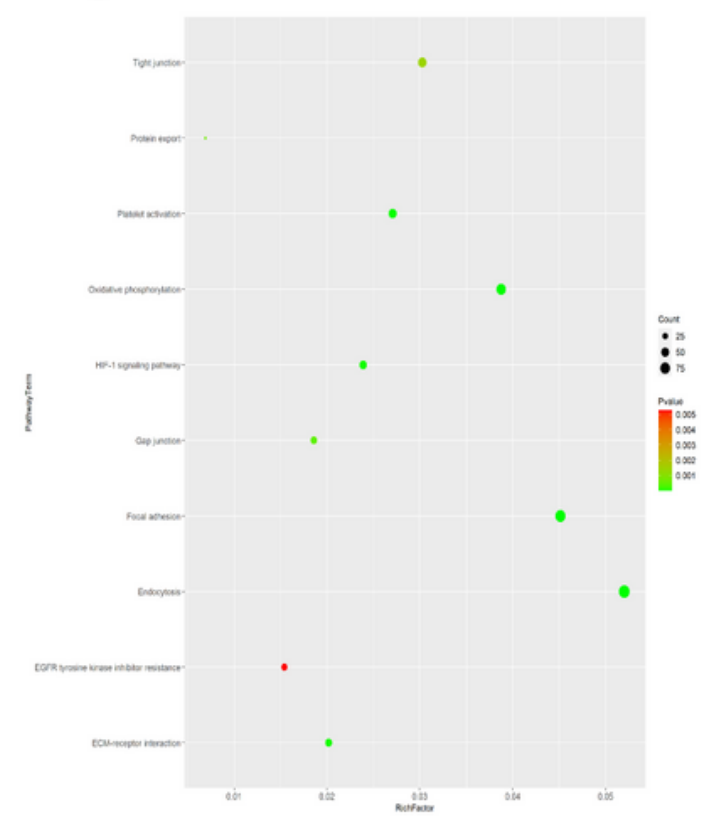


Proteomic analysis of HUMSC and HUCMSC-exos. a Cluster analysis of differentially expressed genes. The colors from green to black and to red represent the expression values of the differentially expressed genes that have increased. Volcano plots for HUCMSCs vs HUCMSC-exos. Statistically significant proteins $(P<0.05)$ with a log2 fold change +2 or -0.5 are represented by red or green dots, respectively. Differentially expressed proteins that did not reach statistical significance are represented by black dots. Selected top GO categories (b) and KEGG pathways (c) enriched in both HUCMSC and HUCMSC-exos. 